\title{
Nitric oxide stimulates human sperm motility via activation of the cyclic GMP/protein kinase G signaling pathway
}

\author{
Erica Miraglia ${ }^{1}$, Federico De Angelis ${ }^{2}$, Elena Gazzano ${ }^{1}$, Hossain Hassanpour $^{3}$, Angela Bertagna ${ }^{4}$, \\ Elisabetta Aldieri ${ }^{1}$, Alberto Revelli ${ }^{2}$ and Dario Ghigo ${ }^{1}$ \\ ${ }^{1}$ Department of Genetics, Biology and Biochemistry, University of Torino, Via Santena, 5/bis, 10126 Torino, Italy, \\ ${ }^{2}$ Reproductive Medicine and IVF Unit, Department of Obstetrical and Gynecological Sciences, S. Anna Hospital, \\ University of Torino, 10126 Torino, Italy, ${ }^{3}$ Department of Basic Sciences, College of Veterinary Medicine, University of \\ Shahrekord, Shahrekord 88186 34141, Iran and ${ }^{4}$ Department of Internal Medicine, University of Torino, S. Giovanni \\ Battista Hospital, 10126 Torino, Italy
}

Correspondence should be addressed to D Ghigo; Email: dario.ghigo@unito.it

\begin{abstract}
Nitric oxide (NO), a modulator of several physiological processes, is involved in different human sperm functions. We have investigated whether NO may stimulate the motility of human spermatozoa via activation of the soluble guanylate cyclase (sGC)/cGMP pathway. Sperm samples obtained by masturbation from 70 normozoospermic patients were processed by the swim-up technique. The kinetic parameters of the motile sperm-rich fractions were assessed by computer-assisted sperm analysis. After a 30-90 min incubation, the NO donor $S$-nitrosoglutathione (GSNO) exerted a significant enhancing effect on progressive motility $(77,78$, and $78 \%$ vs 66,65 , and $62 \%$ of the control at the corresponding time), straight linear velocity $(44,49$, and $48 \mu \mathrm{m} / \mathrm{s}$ vs 34, 35, and $35.5 \mu \mathrm{m} / \mathrm{s})$, curvilinear velocity $(81,83$, and $84 \mu \mathrm{m} / \mathrm{s}$ vs $68 \mu \mathrm{m} / \mathrm{s})$, and average path velocity $(52,57$, and $54 \mu \mathrm{m} / \mathrm{s}$ vs 40,42 , and $42 \mu \mathrm{m} / \mathrm{s})$ at $5 \mu \mathrm{M}$ but not at lower concentrations, and in parallel increased the synthesis of cGMP. A similar effect was obtained with the NO donor spermine NONOate after 30 and $60 \mathrm{~min}$. The GSNO-induced effects on sperm motility were abolished by $1 H$-[1,2,4]oxadiazolo-[4,3-a]quinoxalin-1-one (a specific sGC inhibitor) and mimicked by 8-bromo-cGMP (8-Br-cGMP; a cell-permeating cGMP analog); the treatment with Rp-8-Br-cGMPS (an inhibitor of cGMP-dependent protein kinases) prevented both the GSNO- and the 8-Br-cGMP-induced responses. On the contrary, we did not observe any effect of the cGMP/PRKG1 (PKG) pathway modulators on the onset of hyperactivated sperm motility. Our results suggest that NO stimulates human sperm motility via the activation of sGC, the subsequent synthesis of cGMP, and the activation of cGMP-dependent protein kinases.

Reproduction (2011) 141 47-54
\end{abstract}

\section{Introduction}

Nitric oxide (NO) is a free radical gas, which participates as a mediator in several physiopathological events, such as regulation of vascular tone, neurotransmission, apoptosis, and inflammation (Wink \& Mitchell 1998). $\mathrm{NO}$ is synthesized by NO synthases (NOS), a family of enzymes catalyzing the conversion of L-arginine to L-citrulline and NO with a 1:1 stoichiometry (Nathan \& Xie 1994). Three NOS isoforms have been described: endothelial (eNOS, NOS3), neuronal (nNOS, NOS1), and inducible (iNOS or NOS2; Nathan \& Xie 1994). NO has been demonstrated to play a role in a variety of functions in the human reproductive tract, including sperm motility (Lewis et al. 1996), chemotaxis (Miraglia et al. 2007), and sperm-zona pellucida binding ability (Sengoku et al. 1998). NOS isoforms have been localized in the acrosome and tail of human, mouse, and bovine spermatozoa (Herrero et al. 1996, Meiser \& Schulz 2003), and low motility spermatozoa have been shown to exhibit aberrant patterns of NOS3 immunostaining (O'Bryan et al. 1998). It has been reported that low concentrations of NO (25-100 nM sodium nitroprusside) enhance the motility of human spermatozoa (Hellstrom et al. 1994, Zhang \& Zheng 1996). Accordingly, human sperm motility is inhibited by the NOS inhibitor $\mathrm{N}^{\mathrm{G}}$-nitro-L-arginine methyl ester and by the $\mathrm{NO}$ scavenger methylene blue (Lewis et al. 1996, Donnelly et al. 1997). On the other hand, high NO concentrations (25-125 $\mu \mathrm{M}$ pure NO, 0.25-2.5 mM sodium nitroprusside, $12-600 \mu \mathrm{M} S$-nitroso- $N$-acetylpenicillamine, and 100-125 $\mu \mathrm{M}$ 3-morpholinosydnonimine) seem to exert opposite effects on the motility of human spermatozoa in vitro (Rosselli et al. 1995, Weinberg et al. 1995, Nobunaga et al. 1996). Studies on sperm capacitation showed that NO $(1-100 \mu \mathrm{M}$ spermine NONOate 
(SPNO) or diethylamine-NONOate) increases cAMP levels, thus triggering protein kinase $\mathrm{A}$ activation and tyrosine phosphorylation (Herrero et al. 2000) and is also involved in activation of protein ERKs (Thundathil et al. 2003, O'Flaherty et al. 2006). On the other hand, like in many other cell types, NO activates the soluble guanylate cyclase (sGC) in human spermatozoa (Revelli et al. 2002). The $\mathrm{NO}$ donors sodium nitroprusside and SPNO have been shown to increase the intracellular levels of cGMP in human (Zhang \& Zheng 1996, Revelli et al. 2001) and murine (Herrero et al. 1998) spermatozoa respectively, and recently the sGC has been identified in human sperm by immunoblotting (Willipinski-Stapelfeldt et al. 2004). Although its levels in human sperm are about 100-fold lower than the cAMP content (Willipinski-Stapelfeldt et al. 2004), cGMP has been implicated in several sperm signaling pathways functions, such as capacitation, acrosome reaction, chemotaxis, and sperm-egg interaction (Revelli et al. 2001, 2002, Herrero et al. 2003, Miraglia et al. 2007). cGMP is thought to modulate also sperm motility. Indeed, the cGMP-dependent phosphodiesterase (PDE) inhibitor sildenafil was reported by some authors (Lefievre et al. 2000, Cuadra et al. 2000), but not by others (Andrade et al. 2000, Aversa et al. 2000, Burger et al. 2000), to increase the velocity and amplitude of lateral head displacement in human spermatozoa. Lefievre et al. (2000) observed an inhibition of sperm PDE activity with sildenafil at high concentrations, inhibiting many PDE and causing also an increase in CAMP, whereas Cuadra et al. (2000) reported that sildenafil stimulates sperm motility at much lower concentrations, quite close to the $\mathrm{IC}_{50}$ of sildenafil for the cGMP-dependent PDE. A recent review of ex vivo studies suggests that sildenafil and tadalafil exert a dosedependent effect on sperm motility, which is enhanced at low doses but may be reduced at high concentrations, but further investigations are required to evaluate the mechanisms by which these PDE selective inhibitors modulate sperm motility (Dimitriadis et al. 2008).

Until now no clear data show a direct relationship between exposure to $\mathrm{NO}$, increase of sperm cGMP levels, and changes of human sperm motility. Therefore, the aim of this study is to investigate whether human sperm motility, which is considered one of the most significant fertility-related sperm features (Hirano et al. 2001), is affected by NO via activation of the sGC/cGMP signaling pathway.

\section{Results}

Since the swim-up procedure was performed in sperm washing medium (SWM) containing bicarbonate and albumin, as previously described (Miraglia et al. 2010), for a time sufficient to induce capacitation in most sperm cells, the experiments shown in each point of this paper can be considered as performed on capacitated spermatozoa (see also Materials and Methods section). Preliminary experiments of dose dependence were performed to establish the concentration of the $\mathrm{NO}$ donor $S$-nitrosoglutathione (GSNO) that affects human sperm motility patterns. A progressively motile sperm swims forward in an essentially straight line: rapid progressive motility (A) indicates sperm swimming with a progression velocity $>25 \mu \mathrm{m} / \mathrm{s}$, while slow progressive motility (B) indicates sperm swimming with a progression velocity $=5-25 \mu \mathrm{m} / \mathrm{s}$ (Krause \& Viethen 1999). At the concentration of $5 \mu \mathrm{M}, \mathrm{GSNO}$ exerted a significant enhancing effect on progressive motility ( $A+B$ motility classes) at each time period considered, while at $0.1-1 \mu \mathrm{M}$ it was not effective (Fig. 1A). When the spermatozoa were incubated with $10 \mu \mathrm{M}$ GSNO, progressive motility ( $\mathrm{A}+\mathrm{B}$ classes) was comparable to that of untreated sperm (Fig. 1A). To check how long the effect of $5 \mu \mathrm{M}$ GSNO takes to develop, time-dependence experiments were performed. The increase in sperm motility induced by GSNO was not significant at 10 and
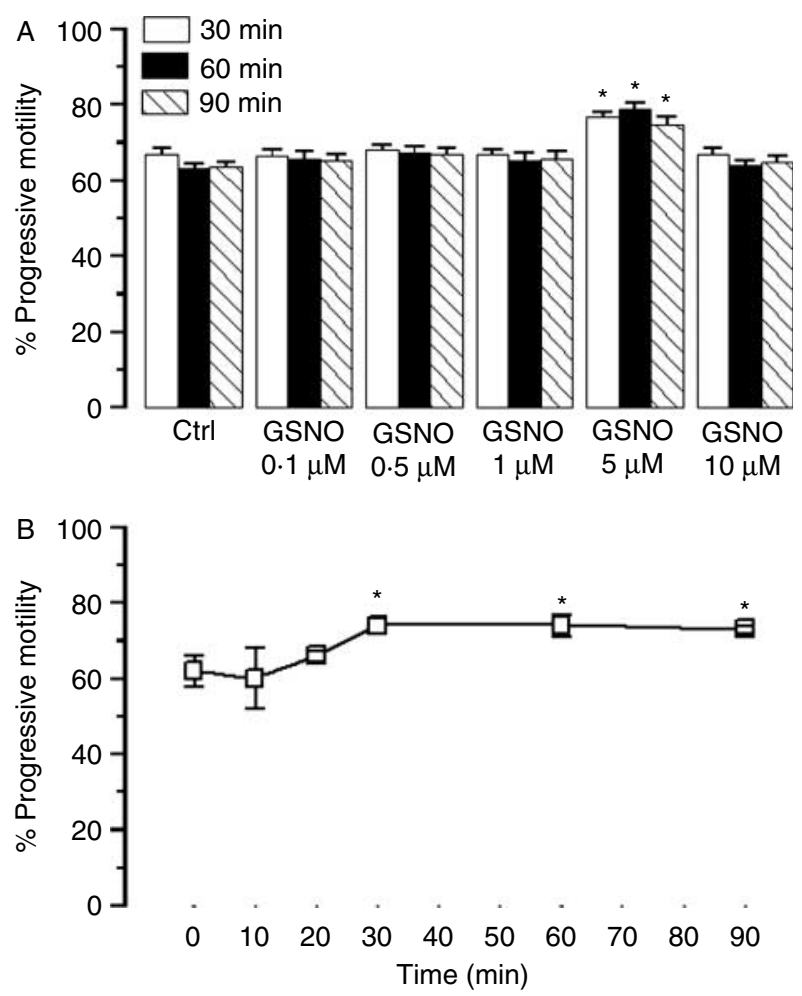

Figure 1 Effect of GSNO on sperm motility patterns. (A) The percentage of spermatozoa exhibiting a forward progressive motility $(A+B$ WHO classes) were recorded by computer-assisted sperm analysis (CASA) after a $30 \mathrm{~min}$ (white bars), 60 min (black bars), or 90 min (hatched bars) incubation of $20 \times 10^{6}$ cells $/ 200 \mu \mathrm{l}$ with $0.1-10 \mu \mathrm{M} S$-nitrosoglutathione (GSNO). All data are presented as means \pm s.E.M. $(n=25)$. Significance versus control at the corresponding incubation time: $* P<0.05$. (B) The percentage of spermatozoa exhibiting a forward progressive motility ( $A+B$ WHO classes) were recorded by CASA after a $10,20,30,60$, or $90 \mathrm{~min}$ incubation of $20 \times 10^{6}$ spermatozoa/200 $\mu \mathrm{l}$ with $5 \mu \mathrm{M}$ GSNO. All data are presented as means \pm s.E.M. $(n=4)$. Significance versus control (CTRL): ${ }^{*} P<0.05$. 
$20 \mathrm{~min}$, but only after an at least 30 min incubation (Fig. 1B). By analyzing each class of motility, we have observed that the increase in progressive motility (WHO classes $A+B$ ) after treatment with GSNO was mainly due to a significant rise in the percentage of $A$ class spermatozoa, which was counterbalanced by a parallel decrease in both $C$ and $D$ class spermatozoa; the amount of spermatozoa exhibiting a B pattern of motility did not change under all the experimental conditions (data not shown). On the contrary, the motion parameters linearity (LIN) and straightness (STR) were unaffected, and no induction of HA was observed (data not shown).

In the same way, GSNO strongly increased the individual parameters of sperm movement straight linear velocity (VSL), curvilinear velocity $(\mathrm{VCL})$, and average path velocity (VAP) when added at $5 \mu \mathrm{M}$ but not at 0.1-1 $\mu \mathrm{M}$ (data not shown). After the incubation with $10 \mu \mathrm{M}$ GSNO, VSL, VCL, and VAP were comparable to those of untreated sperm, thus suggesting that GSNO at this concentration was not yet toxic, but it neither could improve the sperm motility (data not shown). SPNO is a faster NO donor than GSNO, when incubated with several samples $(n=6)$ of spermatozoa, SPNO $0.5 \mu \mathrm{M}$ significantly increased the progressive motility $(A+B$ classes) after 30 (71.2 \pm 2 vs $51 \pm 1 \%$ in controls) and $60 \mathrm{~min}(69 \pm 3$ vs $50 \pm 2 \%$ in controls). In the same experimental conditions, $0.5 \mu \mathrm{M}$ SPNO also increased significantly VSL, VCL, and VAP (data not shown).

Oxidized glutathione (GSSG), the product of GSNO decomposition, is a powerful chelator of copper ions (Singh et al. 1999). Since copper ions can influence the release of $\mathrm{NO}$ from GSNO, we performed further experiments to check whether the increased sperm motility that we observed after incubation with GSNO is due to the chelation of copper by GSSG. We measured sperm motility in the presence of $5 \mu \mathrm{M}$ reduced glutathione (GSH) or GSSG, to exclude that glutathione per se, in any form, could alter the progressive motility; both GSH and GSSG had no significant effect on sperm motility ( $n=4$; data not shown). To chelate the copper, we also performed other experiments with $1 \mathrm{mM}$ EDTA, and even in this case we did not observe any significant modification versus controls and versus GSNO alone ( $n=4$; data not shown). After EDTA treatment the level of calcium was about $1 \mathrm{mM}$.

Thereafter, the $5 \mu \mathrm{M}$ concentration of GSNO was chosen to perform the subsequent experiments. The NO donor induced a significant increase in sperm progressive motility measured by computer-assisted sperm analysis (CASA) after incubation with freshly ejaculated human samples for 30, 60, and $90 \mathrm{~min}$ (Fig. 1B). The sGC inhibitor $1 \mathrm{H}$-[1,2,4] oxadiazolo-[4,3-a]quinoxalin-1-one (ODQ) did not affect the progressive motility when added alone, but completely blunted its GSNO-elicited increase at each time period (Fig. 2). On the other hand, 8-bromo-cGMP (8-Br-cGMP), a cell-permeating cGMP analog, exerted a significant enhancing effect on

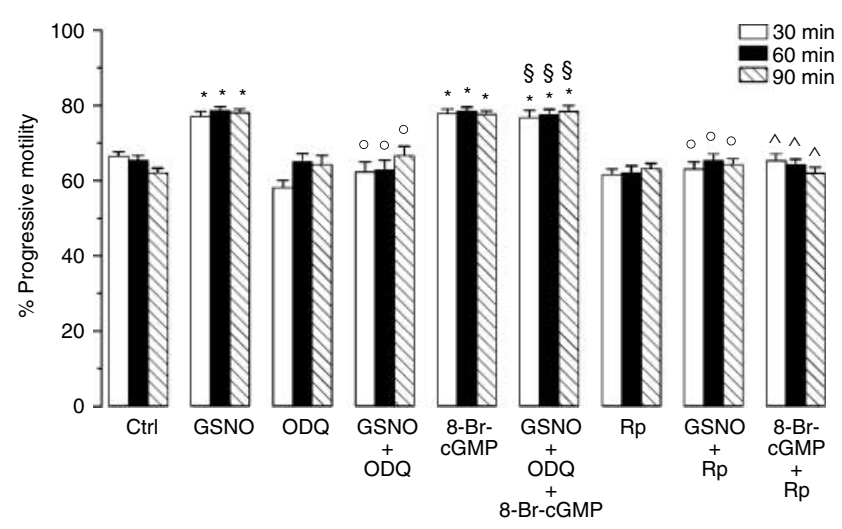

Figure 2 Effect of the modulation of the cGMP pathway on human sperm progressive motility. The forward progressive motility (motility classes $\mathrm{A}+\mathrm{B}$ ) was assessed by CASA in human spermatozoa $\left(20 \times 10^{6} / 200 \mu \mathrm{l}\right)$ incubated for 30,60 , or $90 \mathrm{~min}$ with the following substances, alone or differently combined: $S$-nitrosoglutathione (GSNO, $5 \mu \mathrm{M}), 1 \mathrm{H}$-[1,2,4]oxadiazolo-[4,3-a]quinoxalin-1-one (ODQ, $10 \mu \mathrm{M})$, 8-bromo-cGMP (8-Br-cGMP, $500 \mu \mathrm{M})$, Rp-8-Br-cGMPS (Rp, $10 \mu \mathrm{M})$. All data are presented as means \pm s.E.M. $(n=40)$. Significance versus respective CTRL: ${ }^{*} P<0.001$; versus GSNO: ${ }^{\circ} P<0.001$; versus GSNO+ODQ: ${ }^{\S} P<0.001$; versus 8-Br-CGMP: ${ }^{\wedge} P<0.001$.

progressive motility per se and completely reversed the inhibitory effect of ODQ on the GSNO-stimulated increase (Fig. 2). Finally, the PRKG1 inhibitor Rp-8-Br-cGMPS, which per se did not modify the sperm progressive motility, abolished the effects of GSNO and 8-Br-cGMP on this motion parameter (Fig. 2).

In order to confirm the role of $\mathrm{NO}$ in this process, we also measured the progressive motility in the presence of the NO scavenger PTIO. In all, $100 \mu \mathrm{M}$ 2-phenyl-4,4,5,5,-tetramethylimidazoline-1 oxyl 3-oxide (PTIO) did not affect sperm motility when used alone, but when co-incubated with GSNO $(5 \mu \mathrm{M})$ it completely reversed the increase in motility induced by GSNO (Fig. 3). In the presence of $20 \mu \mathrm{l}$ packed fresh red blood cells, used as reservoirs for the NO scavenger oxyhemoglobin, the motility results were the same observed with PTIO ( $n=3$; data not shown). To this purpose, we incubated the spermatozoa at the reported concentrations used in the other experiments and for the indicated times $(30,60$, and $90 \mathrm{~min}$ ) in the lower compartment of a transwell system (having a polycarbonate transwell insert membrane with pore sizes of $3 \mu \mathrm{m}$, in 24-well plates provided by Corning Incorporated, Apton, MA, USA), the upper compartment containing $20 \mu \mathrm{l}$ packed fresh red blood cells in $0.5 \mathrm{ml}$ SWM. After each incubation time, the upper compartment was taken out and the sperm motility parameters were measured as described in 'Materials and Methods' section.

We also evaluated the effect of $\mathrm{NO}$ on sperm kinetic parameters assessed by CASA. In the presence of GSNO, the VSL markedly increased, an effect that was abolished by ODQ (which per se did not modify this motion parameter), as shown in Fig. 4A; the cGMP analog 


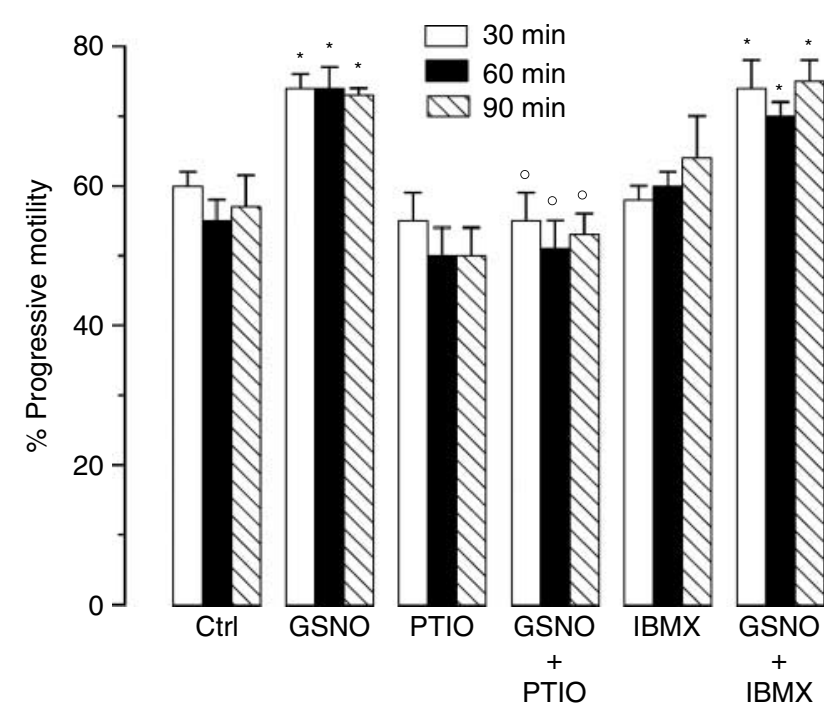

Figure 3 Effect of GSNO, PTIO, and IBMX on sperm motility patterns. The forward progressive motility (motility classes $\mathrm{A}+\mathrm{B}$ ) was assessed by CASA in human spermatozoa $\left(20 \times 10^{6} / 200 \mu \mathrm{l}\right)$ incubated for 30,60 , or 90 min with the following substances, alone or differently combined: $5 \mu \mathrm{M}$ GSNO, $100 \mu \mathrm{M}$ PTIO, and $200 \mu \mathrm{M}$ IBMX. In the case of IBMX, the spermatozoa were pretreated for $20 \mathrm{~min}$ with IBMX before being incubated with $5 \mu \mathrm{M}$ GSNO for 30, 60, or $90 \mathrm{~min}$. All data are presented as means \pm S.E.M. $(n=4)$. Significance versus respective CTRL: $* P<0.05$; versus GSNO: ${ }^{\circ} P<0.05$.

8-Br-cGMP significantly stimulated VSL, and bypassed the inhibition exerted by ODQ on the GSNO-evoked VSL increase (Fig. 4A). The co-incubation with Rp-8Br-cGMPS completely blunted the positive action of both GSNO and 8-Br-cGMP on VSL (Fig. 4A).

The same pattern of response was observed when considering the VCL (Fig. 4B) and the VAP (Fig. 4C) of human spermatozoa treated under the same experimental conditions.

Finally, under the same experimental conditions, GSNO significantly increased the synthesis of cGMP in human spermatozoa at each incubation time considered; the absence of a significant time dependence suggests that GSNO exerts a maximal effect already after $30 \mathrm{~min}$, and that between 30 and $90 \mathrm{~min}$ the synthesis of cGMP is maintained in a steady-state condition. The effect of GSNO was completely abolished by ODQ; as expected, after incubation with 8-Br-cGMP, both alone and together with GSNO and ODQ, the cGMP intracellular level was significantly higher than the control level (Fig. 5). Moreover, in this case, no time dependence was observed, suggesting that in our experimental conditions, the entry of 8 -Br-cGMP into the cells and its degradation were balanced throughout the time of investigation.

Since the measurement of intracellular cGMP was performed in the presence of the PDE inhibitor 3-isobutyl-1-methylxanthine (IBMX) to inhibit cGMP hydrolysis, we performed further motility experiments on samples pretreated for 20 min with $200 \mu \mathrm{M} \mathrm{IBMX}$ and then for 30, 60, and $90 \mathrm{~min}$ with $5 \mu \mathrm{M}$ GSNO. We observed that the pretreatment with IBMX did not influence the enhancement of sperm motility induced by NO (Fig. 3).

\section{Discussion}

The NO/cGMP signaling pathway modulates several physiopathological events of the mammalian reproductive tract (Rosselli et al. 1998). As far as sperm functions are concerned, NO released by sodium nitroprusside has been shown to play an important role in mouse sperm hyperactivation (HA; Herrero et al. 1994) and in

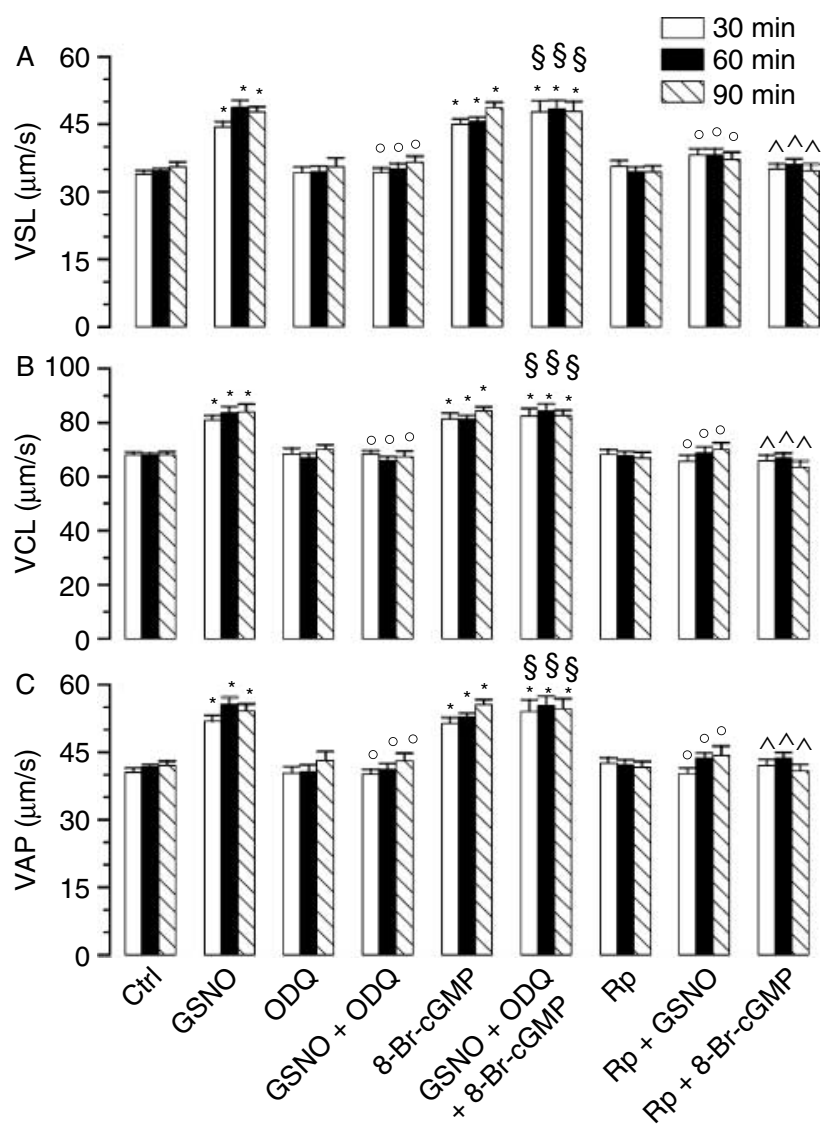

Figure 4 Effects of the modulation of the cGMP pathway on straight linear velocity (VSL, panel A), curvilinear velocity $(\mathrm{VCL}$, panel $\mathrm{B})$, and average path velocity (VAP, panel C) of human spermatozoa. VSL, VCL, and VAP were measured by CASA on human spermatozoa $\left(20 \times 10^{6} / 200 \mu \mathrm{l}\right)$ incubated for 30,60 , or $90 \mathrm{~min}$ in the absence (CTRL) or presence of the following agents, alone or differently combined: $5 \mu \mathrm{M}$ GSNO, $10 \mu \mathrm{M}$ ODQ, $500 \mu \mathrm{M}$ 8-Br-cGMP, and $10 \mu \mathrm{M}$

Rp-8-Br-cGMPS (Rp). Results are shown as means \pm s.E.M. $(n=40)$. (A) Significance versus CTRL: ${ }^{*} P<0.001$; versus GSNO: ${ }^{\circ} P<0.001$; versus GSNO+ODQ: ${ }^{\circledR} P<0.001$; versus 8 -Br-cGMP: ${ }^{\wedge} P<0.001$. (B) Significance versus CTRL: ${ }^{*} P<0.001$; versus GSNO: ${ }^{\circ} P<0.005$; versus GSNO+ODQ: ${ }^{\circledR} P<0.01$; versus 8 -Br-cGMP: ${ }^{\wedge} P<0.005$. (C) Significance versus CTRL: $* P<0.001$; versus GSNO: ${ }^{\circ} P<0.001$; versus GSNO+ODQ: ${ }^{\circledR} P<0.001$; versus 8 -Br-cGMP: ${ }^{\wedge} P<0.001$. 


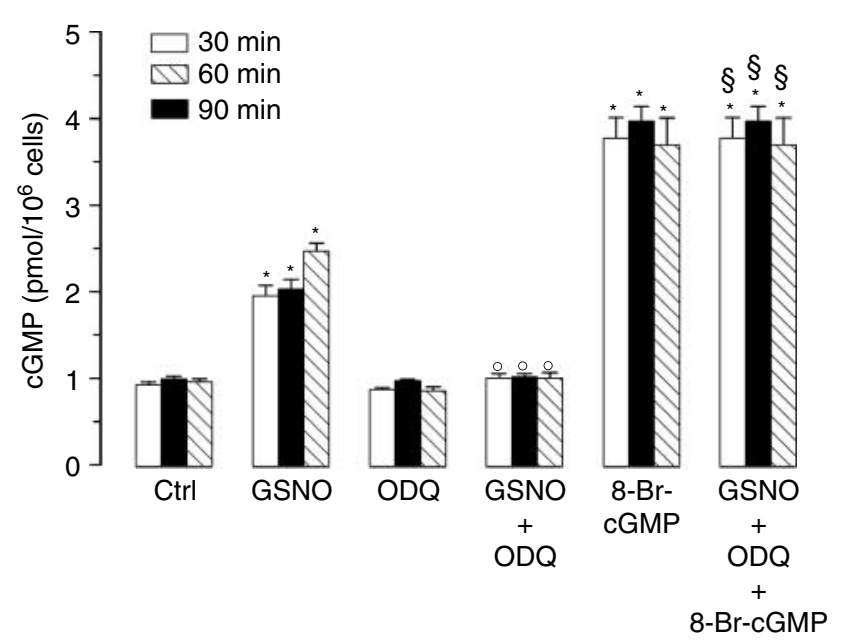

Figure 5 Intracellular cGMP levels in human spermatozoa treated with agents modulating the cGMP pathway. Sperm samples $\left(15 \times 10^{6}\right.$ cells/500 $\mu \mathrm{l}$ ) were pretreated with $200 \mu \mathrm{M}$ IBMX for $20 \mathrm{~min}$, and subsequently they were incubated for 30,60 , or 90 min in the absence (CTRL) or presence of the following substances, alone or in co-incubation: $5 \mu \mathrm{M}$ GSNO, $10 \mu \mathrm{M}$ ODQ, and $500 \mu \mathrm{M}$ 8-Br-cGMP. Then, intracellular cGMP concentration was determined as described in 'Materials and Methods' section. The measurements were performed in triplicate, and data are presented as means \pm s.E.M. $(n=5)$.

Significance versus CTRL: $* P<0.001$; versus GSNO: ${ }^{\circ} P<0.005$; versus GSNO+ODQ: ${ }^{\S} P<0.001$.

the maintenance of postthaw human sperm motility and viability (Hellstrom et al. 1994). Moreover, spermatozoa themselves synthesize NO, and the basal release of this free radical by spermatozoa has been observed to be higher in normozoospermic than in asthenospermic sperm samples; accordingly, normal spermatozoa express more NOS3 and generate more nitrite than spermatozoa of asthenospermic samples (Lewis et al. 1996). Furthermore, the NO scavenger methylene blue and the NOS inhibitor $N^{G}$-nitro-L-arginine methyl ester have been shown to inhibit human sperm motility (Lewis et al. 1996, Donnelly et al. 1997). On the other hand, when female mice null for one of the three NOS isoforms (NOS3, NOS1, and NOS2 respectively) mated with null male mice the rate of IVF was not inhibited (Yang et al. 2005) and this observation does not change the meaning of our results, because in the absence of an NOS isoform, NO can be produced by another isoform. Furthermore, these results were obtained in mice. Finally, in the spermatozoa, NO can be generated as a consequence of a direct hydrogen peroxide attack on arginine (Aitken et al. 2004).

In this work, we provide further evidence suggesting a role for the cGMP signaling pathway in human sperm motility. The NO donor GSNO significantly increased the sperm forward progressive motility after 30-90 min of incubation. GSNO significantly augmented the percentage of A class sperm without modifying the overall amount of B class sperm; it also decreased the percentage of in situ motile (C class) and immotile (D class) cells. In parallel, the NO donor stimulated the sperm kinetic parameters assessed by CASA, VSL, VCL, and VAP. This is in accordance with a previous study reporting that sodium nitroprusside increased human sperm motility (Zhang \& Zheng 1996). Such effect was detectable at 25-100 nM but not at 200-400 nM, whereas we observed a significant motility enhancement using $5 \mu \mathrm{M}$ GSNO. This difference may be due to the different $\mathrm{NO}$ donor used and the different experimental procedure used to measure sperm motility. Indeed, that study evaluated a transmembrane migration ratio (the proportion of human spermatozoa moving across a membrane separating two chambers; Zhang \& Zheng 1996), whereas CASA calculates the percentage of cells exhibiting a forward progressive motility and the kinetic parameters of each cell.

Compared with GSNO (having an half-life of hours, ranging from 10 to $38 \mathrm{~h}$; Nikitovic \& Holmgren 1996, Mancuso et al. 2003), SPNO is a faster NO donor, with a half-life of $39 \mathrm{~min}$ at $37^{\circ} \mathrm{C}$ and $\mathrm{pH} 7.4$ (Keefer et al. 1996). In further experiments using SPNO as NO donor, we observed that also the incubation with SPNO $(0.5 \mu \mathrm{M})$ significantly increased the progressive motility (A + B classes), VSL, VCL, and VAP after 30 and $60 \mathrm{~min}$.

In a previous work, we have demonstrated that GSNO and 8-Br-cGMP exerted a significant chemotactic effect on human spermatozoa without affecting their motion parameters (Miraglia et al. 2007). In that study, both substances were used at different concentrations and time periods compared with those used in this investigation. GSNO exerted a chemoattractant effect at $100 \mathrm{nM}$, while in this study it was ineffective on motility even at $1 \mu \mathrm{M}$. On the other hand, 8-Br-cGMP was used in the previous study at a $1 \mathrm{mM}$ concentration, twofold higher than the one used in the present work. Moreover, in our previous work, we investigated the sperm motion parameters only after 20 min of incubation with GSNO and 8-Br-cGMP, whereas in the present work we used longer (30-90 min) time periods of observation (Miraglia et al. 2007). Since the intracellular levels of cGMP measured after incubation with either GSNO or 8-Br-cGMP were respectively similar in both the experimental works, in spite of the different incubation times and concentrations used, it is likely that these compounds exert a significant effect on sperm motility only when the level of intracellular cGMP is maintained increased for a time longer than the one necessary for cGMP to modulate chemotaxis. This suggestion may make sense, since it is reasonable to assume that at first time sperm needs to be simply oriented versus a source of $\mathrm{NO}$ and only subsequently, when the increase in cGMP shows to be persistently high, the motility should increase.

The effect of GSNO on sperm motility is indeed mediated by an increased synthesis of cGMP, as the sGC inhibitor ODQ blunted the GSNO-elicited motility and abolished the increase in intracellular cGMP induced by 
GSNO. The treatment with the cell-permeating cGMP analog 8-Br-cGMP, which augmented by nearly fourfold the intracellular content of cyclic nucleotide, strongly increased the forward progressive motility and the kinetic parameters VSL, VCL, and VAP. Moreover, 8-Br-cGMP reversed the inhibitory effect of ODQ on the GSNO-evoked increase in progressive motility and velocity, confirming that ODQ inhibited sperm motility by lowering the intracellular level of cGMP.

Taken together, these findings suggest that NO stimulates human sperm motility via the activation of sGC and the subsequent synthesis of cGMP. One of the main targets of cGMP in many tissues is a family of serine/threonine kinases, the PRKG1s (Hofmann 2005). Rp-8-Br-cGMPS, a PRKG1 inhibitor (Kawada et al. 1997), abolished the positive effect exerted by both GSNO and 8-Br-cGMP on sperm motility, suggesting that the effect of endogenous or exogenous cGMP on sperm movement is mediated by PRKG1 activity. Thus, from our data, PRKG1 seems to play a role in mediating not only the NO-elicited chemotaxis and the acrosome reaction (Revelli et al. 2001, Miraglia et al. 2007), but also in modulating several sperm motion patterns. On the other hand, we did not observe any effect of the cGMP/ PRKG1 pathway modulators on the onset of hyperactivated sperm motility.

It is widely acknowledged that spermatozoa in the human female reproductive tract have close and prolonged contact with a significant array of NO-producing cells (Rosselli et al. 1998, Sun et al. 2005, Machado-Oliveira et al. 2008); the exact sites of NO production in the female genital tract remain to be investigated, but Machado-Oliveira et al. (2008) showed that detectable amounts of $\mathrm{NO}$ are produced in human cumulus fragments and oviduct explants. This free radical is relatively unreactive, and is able to diffuse from the cell in which it is generated to the neighbor cells, covering long distances in a very short time (Kröncke et al. 1997). Moreover, spermatozoa themselves produce and release $\mathrm{NO}$ during their trip along the upper female genital tract. This suggests that a complex interaction between spermatozoa, granulosa cells, and other cells of the female reproductive tract may submit human sperm to the amounts of NO that are sufficient to elicit in vivo changes of motility we have observed in vitro.

It is generally accepted that good sperm motility is a major component of normal male fertility. Men with poorly motile or immotile sperm are typically infertile or sterile (Turner 2006). A deeper knowledge of the role of the NO/cGMP/ PRKG1 signaling pathway in the physiopathology of sperm motility could help to pharmacologically improve the fertilization capacity of human sperm or, alternatively, could lead to the development of an effective and safe male contraceptive based on sperm motility impairment.

\section{Materials and Methods}

\section{Reagents}

SWM was supplied by Celbio (Milan, Italy). It is based on the modified human tubal fluid (Quinn et al. 1985), containing sodium bicarbonate (4 mM), HEPES buffer (21 mM), human serum albumin $(5 \mathrm{mg} / \mathrm{ml})$. GSNO, ODQ, 8-Br-cGMP, and IBMX were purchased from Sigma Chemical Co. The inhibitor of cGMP-dependent protein kinases (PRKG1s), 8-bromoguanosine-3', 5'-monophosphorothioate Rp-isomer (Rp-8-Br-cGMPS), was from Biolog Life Science Institute (Bremen, Germany). The $\left[{ }^{3} \mathrm{H}\right] \mathrm{CGMP}$ RIA kit was obtained from Amersham International.

\section{Collection and preparation of sperm samples}

Sperm samples were obtained by masturbation after 3-5 days of sexual abstinence from 70 normozoospermic patients belonging to couples presenting for infertility evaluation. Each donor gave informed consent allowing the use of his semen for our experiments. Institutional review board approval was obtained from the internal ethical committee that authorized the use of semen samples submitted to semen examination for experimental purposes. All samples were allowed to liquefy for at least $30 \mathrm{~min}$ at $37^{\circ} \mathrm{C}$, and then they were evaluated for sperm concentration, motility, and morphology according to the World Health Organization guidelines (World Health Organization 2001). Only specimens with normal parameters (concentration $>20 \times 10^{6}$ spermatozoa $/ \mathrm{ml}$, progressive motility $>50 \%$ ) were used in the experiments.

Motile spermatozoa were capacitated by the swim-up technique $\left(37^{\circ} \mathrm{C}\right.$ for $75 \mathrm{~min}$ in a $5 \% \mathrm{CO}_{2}$ atmosphere) using SWM as previously described (Miraglia et al. 2010). The presence of round cells was initially below $1 \times 10^{6}$ in all sperm samples, and was minimal if not absent after the swim-up technique in the final suspension. After swim-up, the motile sperm-rich fraction was centrifuged at $600 \mathrm{~g}$ for $10 \mathrm{~min}$, the supernatant was discarded, and the pellets were re-suspended in SWM. The concentration of the spermatozoa suspensions was assessed in a Makler counting chamber (Sefi Medical Instruments, Haifa, Israel) under a phase-contrast microscope (magnification $\times 20$ ), and adjusted to $\sim 100 \times 10^{6}$ cells $/ \mathrm{ml}$. The dose-dependent effect of GSNO on sperm motility was investigated in the first 25 samples $\left(20 \times 10^{6}\right.$ cells $\left./ 200 \mu \mathrm{l}\right)$, the effect of the modulation of the cGMP pathway on sperm kinetic parameters was studied in the subsequent 40 samples $\left(20 \times 10^{6}\right.$ cells $/ 200 \mu \mathrm{l}$ ), and finally the ability of the cGMP-modulating agents to modify the intracellular cGMP content was checked in the last 5 samples $\left(15 \times 10^{6}\right.$ cells $\left./ 500 \mu \mathrm{l}\right)$. GSNO was not toxic at the concentrations used, as checked by the eosin $\mathrm{Y}$ exclusion test (Cincik et al. 2007).

\section{Analysis of motility parameters}

Aliquots of sperm suspension $(200 \mu \mathrm{l})$ in SWM, each containing $20 \times 10^{6}$ cells, were incubated under the experimental conditions indicated in section 'Results'. Sperm motility parameters were assessed by CASA 
(CGA-WLJY-9000; CGA Distribution, Florence, Italy) after 30, 60 , and $90 \mathrm{~min}$ of incubation. The following kinetic parameters were measured: percentage of spermatozoa exhibiting a forward progressive motility $(\mathrm{A}+\mathrm{B}$ WHO classes), in situ motility (C WHO class), or no motility (D WHO class); VSL (which represents the average velocity, expressed in $\mu \mathrm{m} / \mathrm{s}$, measured from the beginning to the end of a linear track); VCL (which is the average velocity measured over the actual point-to-point track followed by the cell, expressed as $\mu \mathrm{m} / \mathrm{s}$ ); VAP (corresponding to the average velocity of smoothed cell's pathway, expressed in $\mu \mathrm{m} / \mathrm{s}) ; \mathrm{LIN}=(\mathrm{VSL} / \mathrm{VCL}) \times 100$; STR (the percentage of correspondence of the cell's pathway to a straight line, with $100 \%$ corresponding to the maximal extent of STR; Mortimer 1997). Sperm HA was also considered, using the following parameters: $\mathrm{VCL} \geq 70 \mu \mathrm{m} / \mathrm{s}, \mathrm{ALH} \geq 7 \mu \mathrm{m}$, LIN $\leq 30 \%$, and VSL $\leq 30 \mu \mathrm{m} / \mathrm{s}$ (Green \& Fishel 1999).

\section{Measurement of intracellular cGMP}

The level of intracellular cGMP was measured as previously described (Miraglia et al. 2007). Briefly, aliquots of sperm suspensions $(500 \mu \mathrm{l})$, each containing $15 \times 10^{6}$ cells, were pretreated for 20 min with the PDE inhibitor IBMX $(200 \mu \mathrm{M})$ to inhibit cGMP hydrolysis, and then were co-incubated for 30, 60 , or 90 min with the same substances (GSNO, ODQ, and 8 -Br-cGMP) used for the assessment of motility parameters, alone or differently combined. Subsequently, the samples were centrifuged at $13000 \mathrm{~g}$ for $1 \mathrm{~min}$, the supernatants were discarded, and $50 \mu \mathrm{l}$ absolute ethanol were added to the pellets; ethanol was then evaporated by vacuum centrifugation, and $350 \mu \mathrm{l} \mathrm{Tris/EDTA} \mathrm{buffer} \mathrm{(50} \mathrm{mM} \mathrm{Tris-HCl,} 4$ mM EDTA, pH 7.5) were added. After $10 \mathrm{~min}, 100 \mu \mathrm{l}$ supernatant were tested for the cGMP level with a $\left[^{3} \mathrm{H}\right]$ cGMP immunoassay system. The cGMP content was expressed as pmol/ $/ 10^{6}$ cells. Crossreactivity of the $\left[{ }^{3} \mathrm{H}\right] \mathrm{cGMP}$ immunoassay system with cAMP was $<0.001 \%$.

\section{Statistical analysis}

All data are provided as means \pm s.E.M. The results were analyzed by a one-way ANOVA and Tukey's and Bonferroni's test (software: SPSS 11.0 for Windows; SPSS, Inc., Chicago, IL, USA), including the different times of incubation in the global significance evaluation. A $P$ value $<0.05$ was considered significant.

\section{Declaration of interest}

The authors declare that there is no conflict of interest that could be perceived as prejudicing the impartiality of the research reported.

\section{Funding}

This research was supported by grants from Regione Piemonte (Ricerca Sanitaria Finalizzata) and Ministero dell'Istruzione, dell'Università e della Ricerca, Italy.

\section{References}

Aitken RJ, Ryan AL, Baker MA \& McLaughlin EA 2004 Redox activity associated with the maturation and capacitation of mammalian spermatozoa. Free Radical Biology \& Medicine 36 994-1010. (doi:10. 1016/j.freeradbiomed.2004.01.017)

Andrade JR, Traboulsi A, Hussain A \& Dubin NH 2000 In vitro effects of sildenafil and phentolamine, drugs used for erectile dysfunction, on human sperm motility. American Journal of Obstetrics and Gynecology 182 1093-1095. (doi:10.1067/mob.2000.105406)

Aversa A, Mazzilli F, Rossi T, Delfino M, Isidori AM \& Fabbri A 2000 Effects of sildenafil (Viagra) administration on seminal parameters and postejaculatory refractory time in normal males. Human Reproduction $\mathbf{1 5}$ 131-134. (doi:10.1093/humrep/15.1.131)

Burger M, Sikka SC, Bivalacqua TJ, Lamb DJ \& Hellstrom WJ 2000 The effect of sildenafil on human sperm motion and function from normal and infertile men. International Journal of Impotence Research 12 229-234. (doi:10.1038/sj.ijir.3900551)

Cincik M, Ergur AR, Tutuncu L, Muhcu M, Kilic M, Balaban B \& Urman B 2007 Combination of hypoosmotic swelling/eosin $Y$ test for sperm membrane integrity evaluation: correlations with other sperm parameters to predict ICSI cycles. Archives of Andrology 53 25-28. (doi:10.1080/ 01485010600888912)

Cuadra DL, Chan PJ, Patton WC, Stewart SC \& King A 2000 Type 5 phosphodiesterase regulation of human sperm motility. American Journal of Obstetrics and Gynecology 182 1013-1015. (doi:10.1067/ mob.2000.105435)

Dimitriadis F, Giannakis D, Pardalidis N, Zikopoulos K, Paraskevaidis E, Giotitsas N, Kalaboki V, Tsounapi P, Baltogiannis D, Georgiou I et al. 2008 Effects of phosphodiesterase-5 inhibitors on sperm parameters and fertilizing capacity. Asian Journal of Andrology 10 115-133. (doi:10. 1111/j.1745-7262.2008.00373.x)

Donnelly ET, Lewis SEM, Thompson W \& Chakravarthy U 1997 Sperm nitric oxide and motility: the effects of nitric oxide synthase stimulation and inhibition. Molecular Human Reproduction 3 755-762. (doi:10. 1093/molehr/3.9.755)

Green S \& Fishel S 1999 Morphology comparison of individually selected hyperactivated and non-hyperactivated human spermatozoa. Human Reproduction 14 123-130. (doi:10.1093/humrep/14.1.123)

Hellstrom WJG, Bell M, Wang R \& Sikka SC 1994 Effects of sodium nitroprusside on sperm motility, viability and lipid peroxidation. Fertility and Sterility 61 1117-1122.

Herrero MB, Cebral E, Boquet M, Viggiano JM, Vitullo A \& Gimeno MA 1994 Effect of nitric oxide on mouse sperm hyperactivation. Acta Physiologica, Pharmacologica et Therapeutica Latinoamericana 44 65-69.

Herrero MB, Perez Martinez S, Viggiano JM, Polak JM \& de Gimeno MF 1996 Localization by indirect immunofluorescence of nitric oxide synthase in mouse and human spermatozoa. Reproduction, Fertility, and Development 8 931-934. (doi:10.1071/RD9960931)

Herrero MB, Cebral E, Franchi A, Motta A \& Gimeno MF 1998 Progesterone enhances prostaglandin $E_{2}$ production via interaction with nitric oxide in the mouse acrosome reaction. Biochemical and Biophysical Research Communications 252 324-328. (doi:10.1006/ bbrc.1998.9638)

Herrero MB, Chatterjee S, Lefièvre L, de Lamirande E \& Gagnon C 2000 Nitric oxide interacts with the CAMP pathway to modulate capacitation of human spermatozoa. Free Radical Biology \& Medicine 29 522-536. (doi:10.1016/S0891-5849(00)00339-7)

Herrero MB, de Lamirande E \& Gagnon C 2003 Nitric oxide is a signaling molecule in spermatozoa. Current Pharmaceutical Design 9 419-425. (doi:10.2174/1381612033391720)

Hirano Y, Shibahara H, Obara H, Suzuki T, Takamizawa S, Yamaguchi C, Tsunoda H \& Sato I 2001 Relationships between sperm motility characteristics assessed by the computer-aided sperm analysis (CASA) and fertilization rates in vitro. Journal of Assisted Reproduction and Genetics 18 213-218. (doi:10.1023/A:1009420432234)

Hofmann F 2005 The biology of cyclic GMP-dependent protein kinases. Journal of Biological Chemistry 280 1-4. (doi:10.1074/jbc.R400035200)

Kawada T, Toyosato A, Islam MO, Yoshida Y \& Imai S 1997 cGMP-kinase mediates CGMP- and cAMP-induced $\mathrm{Ca}^{2+}$ desensitization of skinned rat artery. European Journal of Pharmacology 323 75-82. (doi:10.1016/ S0014-2999(97)00028-9) 
Keefer LK, Nims RW, Davies KM \& Wink DA 1996 "NONOates" (1-substituted diazen-1-ium-1,2-diolates) as nitric oxide donors: convenient nitric oxide dosage forms. Methods in Enzymology 268 281-293. (doi:10.1016/S0076-6879(96)68030-6)

Krause W \& Viethen G 1999 Quality assessment of computer-assisted semen analysis (CASA) in the andrology laboratory. Andrologia 31 125-129. (doi:10.1111/j.1439-0272.1999.tb01398.x)

Kröncke KD, Fehsel K \& Kolb-Bachofen V 1997 Nitric oxide: cytotoxicity versus cytoprotection - how, why, when, and where? Nitric Oxide $\mathbf{1}$ 107-120. (doi:10.1006/niox.1997.0118)

Lefievre L, DeLamirande E \& Gagnon C 2000 The cyclic GMP-specific phosphodiesterase inhibitor, sildenafil, stimulates human sperm motility and capacitation but not acrosome reaction. Journal of Andrology $\mathbf{2 1}$ 929-937.

Lewis SEM, Donnelly ET, Sterling ESL, Kennedy MS, Thompson W \& Chakravarthy U 1996 Nitric oxide synthase and nitrite production in human spermatozoa: evidence that endogenous nitric oxide is beneficial to sperm motility. Molecular Human Reproduction 2 873-878. (doi:10. 1093/molehr/2.11.873)

Machado-Oliveira G, Lefievre L, Ford C, Herrero MB, Barratt C, Connolly TJ, Nash K, Morales-Garcia A, Kirkman-Brown J \& Publicover S 2008 Mobilisation of $\mathrm{Ca}^{2+}$ stores and flagellar regulation in human sperm by $S$-nitrosylation: a role for $\mathrm{NO}$ synthesised in the female reproductive tract. Development 135 3677-3686. (doi:10.1242/dev.024521)

Mancuso C, Bonsignore A, Di Stasio E, Mordente A \& Motterlini R 2003 Bilirubin and $S$-nitrosothiols interaction: evidence for a possible role of bilirubin as a scavenger of nitric oxide. Biochemical Pharmacology $\mathbf{6 6}$ 2355-2363. (doi:10.1016/j.bcp.2003.08.022)

Meiser H \& Schulz R 2003 Detection and localization of two constitutive NOS isoforms in bull spermatozoa. Anatomia, Histologia, Embryologia 32 321-325. (doi:10.1111/j.1439-0264.2003.00459.x)

Miraglia E, Rullo ML, Bosia A, Massobrio M, Revelli A \& Ghigo D 2007 The stimulation of nitric oxide/cyclic GMP signaling pathway elicits human sperm chemotaxis in vitro. Fertility and Sterility 87 1059-1063. (doi:10. 1016/j.fertnstert.2006.07.1540)

Miraglia E, Lussiana C, Viarisio D, Racca C, Cipriani A, Gazzano E, Bosia A, Revelli A \& Ghigo D 2010 The pentose phosphate pathway plays an essential role in supporting human sperm capacitation. Fertility and Sterility 93 2437-2440. (doi:10.1016/j.fertnstert.2009.09.005)

Mortimer ST 1997 A critical review of the physiological importance and analysis of sperm movement in mammals. Human Reproduction Update 3 403-439. (doi:10.1093/humupd/3.5.403)

Nathan C \& Xie QW 1994 Nitric oxide synthases: roles, tolls, and controls. Cell 78 915-918. (doi:10.1016/0092-8674(94)90266-6)

Nikitovic D \& Holmgren A 1996 S-nitrosoglutathione is cleaved by the thioredoxin system with liberation of glutathione and redox regulating nitric oxide. Journal of Biological Chemistry 271 19180-19185. (doi:10. 1074/jbc.271.32.19180)

Nobunaga T, Tokugawa Y, Hashimoto K, Kubota Y, Sawai K, Kimura T, Shimoya K, Takemura M, Matsuzaki N, Azuma C et al. 1996 Elevated nitric oxide concentration in the seminal plasma of infertile males: nitric oxide inhibits sperm motility. American Journal of Reproductive Immunology 36 193-197.

O'Bryan MK, Zini A, Cheng CY \& Schlegel PN 1998 Human sperm endothelial nitric oxide synthase expression: correlation with sperm motility. Fertility and Sterility 70 1143-1147. (doi:10.1016/S00150282(98)00382-3)

O'Flaherty C, de Lamirande E \& Gagnon C 2006 Reactive oxygen species modulate independent protein phosphorylation pathways during human sperm capacitation. Free Radical Biology \& Medicine 40 1045-1055. (doi:10.1016/j.freeradbiomed.2005.10.055)

Quinn P, Kerin JF \& Warnes GM 1985 Improved pregnancy rate in human in vitro fertilization with the use of a medium based on the composition of human tubal fluid. Fertility and Sterility 44 493-498.
Revelli A, Costamagna C, Moffa F, Aldieri E, Ochetti S, Bosia A, Massobrio M, Lindblom B \& Ghigo D 2001 Signaling pathway of nitric oxide-induced acrosome reaction in human spermatozoa. Biology of Reproduction 64 1708-1712. (doi:10.1095/biolreprod64.6.1708)

Revelli A, Ghigo D, Moffa F, Massobrio M \& Tur-Kaspa I 2002 Guanylate cyclase activity and sperm function. Endocrine Reviews 23 484-494. (doi:10.1210/er.2001-0020)

Rosselli M, Dubey RK, Imithurn B, Macas E \& Keller PJ 1995 Effects of nitric oxide on human spermatozoa: evidence that nitric oxide decreases sperm motility and induces sperm toxicity. Human Reproduction $\mathbf{1 0}$ 1786-1790.

Rosselli M, Keller PJ \& Dubey RK 1998 Role of nitric oxide in the biology, physiology and pathophysiology of reproduction. Human Reproduction Update 4 3-24. (doi:10.1093/humupd/4.1.3)

Sengoku K, Tamate K, Yoshida T, Takaoka Y, Miyamoto T \& Ishikawa M 1998 Effects of low concentrations of nitric oxide on the zona pellucida binding ability of human spermatozoa. Fertility and Sterility 69 522-527. (doi:10.1016/S0015-0282(97)00537-2)

Singh RJ, Hogg N, Goss SPA, Antholine WE \& Kalyanaraman B 1999 Mechanism of superoxide dismutase/ $\mathrm{H}_{2} \mathrm{O}_{2}$-mediated nitric oxide release from S-nitrosoglutathione - role of glutamate. Archives of Biochemistry and Biophysics 372 8-15. (doi:10.1006/abbi.1999.1447)

Sun F, Bahat A, Gakamsky A, Girsh E, Katz N, Giojalas LC, Tur-Kaspa I \& Eisenbach M 2005 Human sperm chemotaxis: both the oocyte and its surrounding cumulus cells secrete sperm chemoattractants. Human Reproduction 20 761-767. (doi:10.1093/humrep/deh657)

Thundathil J, de Lamirande E \& Gagnon C 2003 Nitric oxide regulates the phosphorylation of the threonine-glutamine-tyrosine motif in proteins of human spermatozoa during capacitation. Biology of Reproduction $\mathbf{6 8}$ 1291-1298. (doi:10.1095/biolreprod.102.008276)

Turner RM 2006 Moving to the beat: a review of mammalian sperm motility regulation. Reproduction, Fertility, and Development 18 25-38. (doi:10. 1071/RD05120)

Weinberg JB, Doty M, Bonaventura J \& Haney AF 1995 Nitric oxide inhibition of human sperm motility. Fertility and Sterility 64 408-413.

Willipinski-Stapelfeldt B, Lubberstedt J, Stelter S, Vogt K, Mukhopadhyay AK \& Muller D 2004 Comparative analysis between cyclic GMP and cyclic AMP signalling in human sperm. Molecular Human Reproduction 10 543-552. (doi:10.1093/molehr/gah065)

Wink DA \& Mitchell JB 1998 Chemical biology of nitric oxide: insights into regulatory, cytotoxic, and cytoprotective mechanisms of nitric oxide. Free Radical Biology \& Medicine 25 434-456. (doi:10.1016/S08915849(98)00092-6)

World Health Organization 2001 Laboratory Manual for the Examination of Human Semen and Sperm-Cervical Mucus Interaction. Cambridge: Cambridge University Press.

Yang JZ, Ajonuma LC, Rowlands DK, Tsang LL, Ho LS, Lam SY, Chen WY, Zhou CX, Chung YW, Cho CY et al. 2005 The role of inducible nitric oxide synthase in gamete interaction and fertilization: a comparative study on knockout mice of three NOS isoforms. Cell Biology International 29 785-791. (doi:10.1016/j.cellbi.2005.05.005)

Zhang H \& Zheng RL 1996 Possible role of nitric oxide on fertile and asthenozoospermic infertile human sperm functions. Free Radical Research 25 347-354. (doi:10.3109/10715769609149057)

Received 22 March 2010

First decision 15 April 2010

Revised manuscript received 22 September 2010

Accepted 20 October 2010 